\title{
Primary response of the sGC heme binding domain to the cleavage of the Fe-His bond
}

\author{
Huali Zhang ${ }^{1}$, Ming Lu', Yuebin Zhang', Zhengqiang $\mathrm{Li}^{1}{ }^{1}$ * \\ ${ }^{1}$ Key Laboratory for Molecular Enzymology and Engineering of the Ministry of Education (Jilin University), 130021 Changchun, Jilin
} (P. R. China); Zhengqiang Li* - E-mail: lzq@jlu.edu.cn; Fax: 86431 88499261; * Corresponding author

received February 29, 2008; revised March 24, 2008; accepted March 25, 2008; published April 11, 2008

\begin{abstract}
:
Soluble guanylate cyclase (sGC) is an important heme sensor protein. Regulation of the status of heme in the heme binding domain (or HNOX domain) by various gaseous activators can increase the catalytic efficiency of the cyclase domain. Several studies have demonstrated that the full activation of sGC is directly related to the cleavage of the Fe-His bond of the HNOX domain. To expand the primary response of the SGC HNOX domain to the cleavage event, a structural model of the sGC HNOX domain was constructed using homology modeling and the Fe-His bond was released at $6 \mathrm{~ns}$ of a 10-ns molecular dynamics simulation. An instant increment of C $\alpha$-RMSD over L2 (Loop2, residues 124-130) was found after the cleavage of the Fe-His bond, which was consistent with the principle component analysis (PCA). The energy analysis results suggest that the motions of L2 are energetic. Based on the results, energetic conformational transformation of L2 is identified as the primary response of the sGC HNOX domain to the cleavage of the Fe-His bond.
\end{abstract}

Keywords: soluble guanylate cyclase; information transmission; HNOX domain; homology modeling; molecular dynamics

\section{Background:}

In general, the functional heme sensor proteins contain both the heme binding domain and the catalytic domain. The transmission of the binding signals of the heme moiety induced by various gaseous activators from the heme binding domain to the catalytic domain is essential for regulation of protein activity. Soluble guanylate cyclase (sGC), a significant heme sensor protein, catalyzes the conversion of GTP to cGMP in vivo. The increase of intracellular cGMP mediated by $\mathrm{sGC}$ is related to many physiological processes including cellular growth and contractility, cardiovascular homeostasis, inflammation, sensory transduction, and neuronal plasticity and learning. Mammalian sGC is a heterodimeric heme enzyme composed of one $\alpha$ subunit and one $\beta$ subunit. In the native enzyme, the heme prosthetic group is bound to the sGC heme binding domain (or heme nitric oxide and/or oxygen binding domain, HNOX domain) of the $\beta$ subunit via the coordinated bond with $\beta 105$-His and is stabilized through the interaction of its propionic acid groups with Y135, S137 and R139 residues of the $\beta$ subunit [1]. The C-terminal regions of the two subunits make up the cyclase domain (or catalytic domain). The binding of $\mathrm{NO} / \mathrm{CO}$ to the heme moiety of the HNOX domain can increase the catalytic efficiency of the cyclase domain [1], and the cleavage of the Fe-His bond is the key event related to the full activation of $\mathrm{sGC}[2,3]$. However, the mechanism through which the activation information is ISSN 0973-2063

Bioinformation 2(7): 296-300 (2008) propagated from the HNOX domain to the cyclase domain is still unclear.

The sGC HNOX domain possesses heme characteristics similar to those of the full-length enzyme during the binding process of gaseous activators $[4,5]$. Elucidating the primary response of the sGC HNOX domain to the cleavage of the Fe-His bond should be helpful in clarifying the activation information transmission pathway in sGC.

HNOX domains observed in a number of proteins in bacteria and animals were identified as homologous to the sGC HNOX domain [6]. Hitherto, only two HNOX domains have been crystallized $[7,8]$, and the Ns HNOX domain is the one sharing the higher sequence identity with the sGC HNOX domain. Remarkably, the Ns HNOX has ligand binding properties similar to those of sGC [9]. The Ns HNOX domain, sharing 35\% sequence identity with the sGC HNOX domain, was also postulated to be evolutionarily homologous to the sGC HNOX domain [6]. These observations imply that the crystal structures of the Ns HNOX domain should be the best template for homology modeling of the sGC HNOX domain. In this work, a structural model of the sGC HNOX domain (SMSH) was constructed using homology modeling based on the recently published crystal structure of a HNOX domain from Nostoc sp (Ns 
HNOX domain). A 10-ns molecular dynamics (MD) simulation, starting from the energy-minimized structure of SMSH, was applied to provide molecular-level insights into the primary response of the sGC HNOX domain to the cleavage of the Fe-His bond. The energetic conformational transformation of L2 is identified as the primary response of the sGC HNOX domain to the cleavage event.

\section{Methodology:}

Generation of a homology model of the sGC HNOX domain

A sequence alignment of the sGC HNOX domain (gi: 118056, 1-184) and the Ns HNOX (gi: 17229770, 1-182) was generated using T-coffee [10] and was further revised considering the secondary structure and the conservation of HNOX domains (Figure 1). A structural model of the sGC HNOX domain (SMSH) was constructed using the MODELLER package [11], through comparison with the crystal structure of the Ns HNOX domain (resolution: 2.5, PDB ID: 2O0G). The DOPE scores, $\varphi-\psi$ angle disturbution and G-scores were used in the model selection process. The heme group was docked onto the structural model using the MODELLER package as well, by comparing the relative position of the heme group in the Ns HNOX domain.

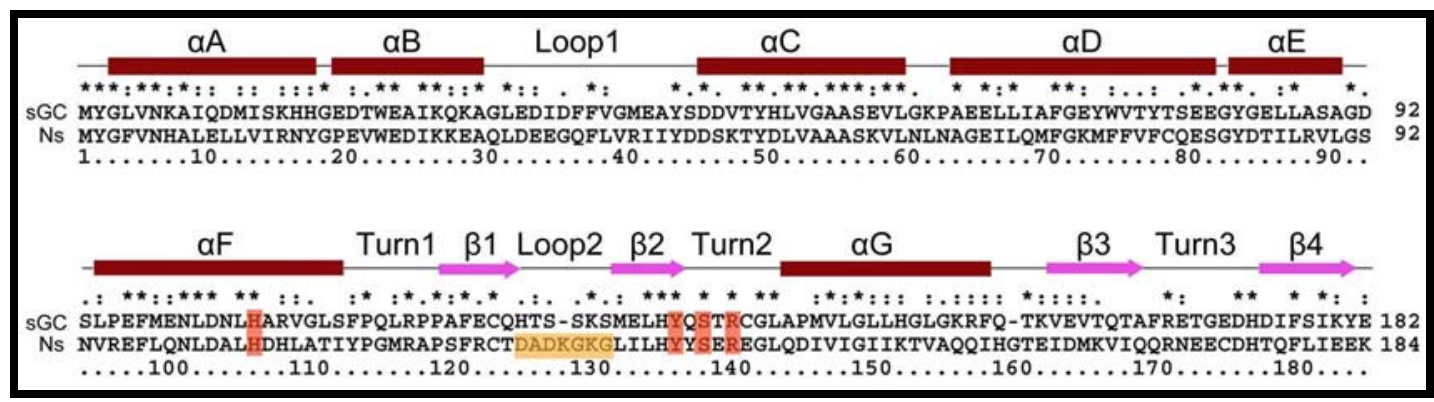

Figure 1: The alignment of the sGC HNOX domain (gi: 118056) with the Ns HNOX domain (gi: 17229770). The structural elements ( $\alpha$-helix, $\beta$-sheet, turn and loop) are labeled. H105, Y135, S137, R139 and the residues of L2 (Loop2, residues 124-130) are highlighted.

\section{MD calculation}

The SMSH was in a six-coordinate state using $\mathrm{CO}$ as the distal ligand and His105 as the proximal ligand. Histidine $\mathrm{N} \delta$ was protonated with the Psfgen plug-in of the VMD program [12]. The model was solvated in a $10 \AA$ cubic water box. The VMD plug-in Autoionize was used to add $\mathrm{Na}^{+}$and $\mathrm{Cl}^{-}$ions at a concentration of $50 \mathrm{mM}$ to neutralize the net charge of the unit cell. The MD simulation was performed using the parallel molecular dynamics program NAMD 2.6 [13] along with the CHARMM22 force field for protein and heme as well as the TIP3P model for water. The simulation was carried out at a temperature of $310 \mathrm{~K}$ and a pressure of 1 bar (NPT ensemble) with periodic boundary condition. The integration time step was $1.0 \mathrm{fs}$. The particle-mesh Ewald (PME) method generated a full electrostatic evaluation. The entire system was minimized and equilibrated with the following stages: (1) 10-ps minimization with fixed backbone atoms; (2) 10-ps minimization with constrained backbone atoms; (3) 50ps Langevin dynamics heating the system from 0 to 310 $\mathrm{K}$ with fixed backbone atoms; (4) 200-ps equilibration of water solvent with fixed backbone atoms; (5) 750-ps equilibration with $\mathrm{C} \alpha$ constrained. Then the equilibrated system was used for a 9-ns MD simulation. During all stages, the distances from Tyr135, Ser137 and Arg139 to the heme propionate groups (D1: $\mathrm{HH}_{135}-\mathrm{O} 1 \mathrm{D}_{\text {heme, }} \mathrm{D} 2$ :

ISSN 0973-2063

Bioinformation 2(7): 296-300 (2008)
$\left.\mathrm{HH}_{137}-\mathrm{O} 2 \mathrm{D}_{\text {heme }}, \mathrm{D} 3: \mathrm{NH}_{139}-\mathrm{O} 1 \mathrm{~A}_{\text {heme }}\right)$ were constrained. During the last $4 \mathrm{~ns}$, the Fe-His bond was released to trigger the conformational transmission. The atomic coordinates were saved every 1 ps. The trajectory visualization and analysis, including energy calculations, were all accomplished with VMD programs.

Principal component analysis [14]

The eigenvectors and eigenvalues of the covariance matrix of $\mathrm{C} \alpha$ positional deviations were calculated using the NumPy module of the Python program. The projections of $\mathrm{C} \alpha$ motions onto the first eigenvector were calculated by the formula below:

$\mathrm{P}=\left(\mathrm{q} \times \mathrm{q}^{\mathrm{T}} \times \mathrm{M}^{\mathrm{T}}\right)+\mathrm{N}$

where $\mathrm{q}$ is the first eigenvector, $\mathrm{M}$ is the Cartesian coordinates matrix of $\mathrm{C} \alpha$ positional deviations, $\mathrm{N}$ is the Cartesian coordinates matrix of $\mathrm{C} \alpha$ atoms, $\mathrm{P}$ is the Cartesian coordinates matrix of $\mathrm{C} \alpha$ atoms reflecting $\mathrm{C} \alpha$ atoms essential motions. The configurations based on Cartesian coordinates of matrix $\mathrm{P}$ were visualized with VMD programs.

\section{Discussion:}

Homology model of the sGC HNOX domain

A structural model of the sGC HNOX domain (SMSH) was constructed. From the Ramachandran plot statistics of SMSH, the models were found to have good
Bioinformation, an open access forum (C) 2008 Biomedical Informatics Publishing Group 
stereochemistry, with $95.8 \%$ of residues in the most favored region. The G-score computed by Procheck is 0.08 . After molecular minimization, the models retained good stereochemistry, with $90.7 \%$ of residues in the most favored region and the G-scores is -0.14 . All the Gscores computed by Procheck are above -0.5 . The result indicated the structure of SMSH is well defined. The SMSH fold consists of seven $\alpha$-helices, and one fourstranded anti-parallel $\beta$-sheet ( $\beta 1-4)$. The structural elements including $\alpha$-helices, $\beta$-sheets, turns and loops are labeled in Figure 2a.

\section{MD simulation of SMSH}

The binding of NO to the heme of sGC can induce the clevage of the Fe-His bond, which results in a 200-400fold enhancement of sGC catalytic ability [1]. The synergistic activation of $\mathrm{sGC}$ by $\mathrm{CO}$ and $\mathrm{YC}-1$ also involves the cleavage of the Fe-His bond [2, 3]. These data indicate that the cleavage of the Fe-His bond is essential to the full activation of sGC. To investigate the primary response of SMSH to the cleavage of the Fe-His bond, the Fe-His bond was released during the MD simulation to trigger the conformational change of SMSH. The propionate hydrogen network bond has been found essential for transmitting the activation information from heme to the heme binding and catalytic domains of the heme sensor protein [15]. In SMSH, Y135, S137 and R139 residues are involved in the formation of the propionate hydrogen network (Figure 2a). Y135, S137 and R139 residues have been found important for the binding of heme to the sGC HNOX domain [16, 17]. The studies on the Ns HNOX domain also suggested that Y135, S137 and R139 residues are strongly bound to the propionate groups during the heme pivot-bend process [8]. Thus, in our MD simulation of $\mathrm{SMSH}$, the hydrogen network was constrained to keep the stable binding of heme and the integrity of the information transmission pathway.

The trajectory was started from the energy-minimized structure of SMSH. The Fe-His bond was released at 6 ns of the simulation time. The RMSD of the simulation are stable after $1 \mathrm{~ns}$ and the $\mathrm{C} \alpha$-RMSD oscillated between 1.5 and $2.0 \AA$. The average radius of gyration $(\mathrm{Rg})$ of $\mathrm{C} \alpha$ atoms over $2-6 \mathrm{~ns}$ is $16.83 \AA( \pm 0.08)$, and the average $\mathrm{Rg}$ of $\mathrm{C} \alpha$ atoms over $4-10 \mathrm{~ns}$ is $16.83 \AA( \pm 0.08)$ as well. These data indicate that the overall stability of SMSH is not affected by the cleavage event. The structural element L2 exhibited a remarkable increment by $\sim 1.5 \AA$ during 6-6.5 $\mathrm{ns}$ of simulation time. Time-resolved crystallography and UV resonance Raman (UVRR) spectroscopy studies on myoglobin suggested that the primary response of the protein to the conformation change of the heme group was completed in less than 150 ps $[18, \mathbf{1 9}$. The molecular size of SMSH is similar to myoglobin and the structural transformation of $\mathrm{L} 2$ happens in 500 ps after the release of the Fe-His bond (Figure 2). This evidence suggests that the structural transformation of L2 is instant and remarkable response of SMSH to the cleavage of the Fe-His bond.

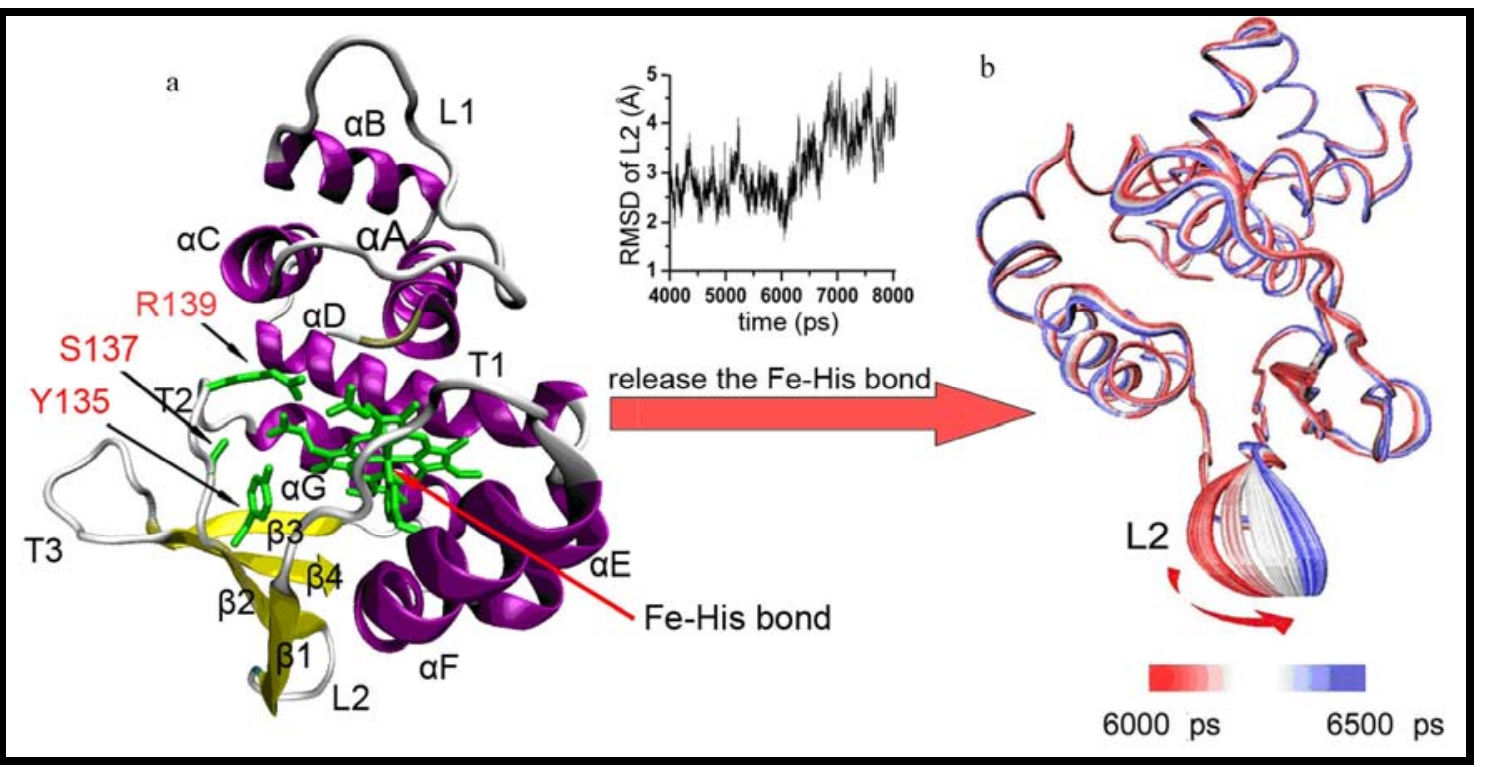

Figure 2: The primary conformational changes of SMSH upon release of the Fe-His bond during MD simulation. (a) Model showing the overall fold of SMSH. Purple color indicates $\alpha$-helix structure and yellow color indicates $\beta$-sheet structure; (b) Superposition of 50 configurations obtained by projecting the $\mathrm{C} \alpha$ motion over 6-6.5 ns onto the first eigenvector, each separated by $10 \mathrm{ps}$. 


\section{www.bioinformation.net}

We have calculated the protein potential energy during the 10-ns simulation. The release of the Fe-His bond results in an increment of the protein potential energy by $150-190 \mathrm{kcal} / \mathrm{mol}$ during 6-6.5 ns. Interestingly, the increase of protein potential energy and the increase of $\mathrm{C} \alpha$-RMSD of L2 happen during the same time period. The conformational changes of L2 are coupled with an interaction energy increment between L2 and other structural elements by $90-120 \mathrm{kcal} / \mathrm{mol}$ during $6-6.5 \mathrm{~ns}$ (Figure 3). This evidence indicates that the increment of protein potential energy during 6-6.5 ns is mainly attributed to the interaction between $\mathrm{L} 2$ and other structural elements, and the motions of L2 are energetic.

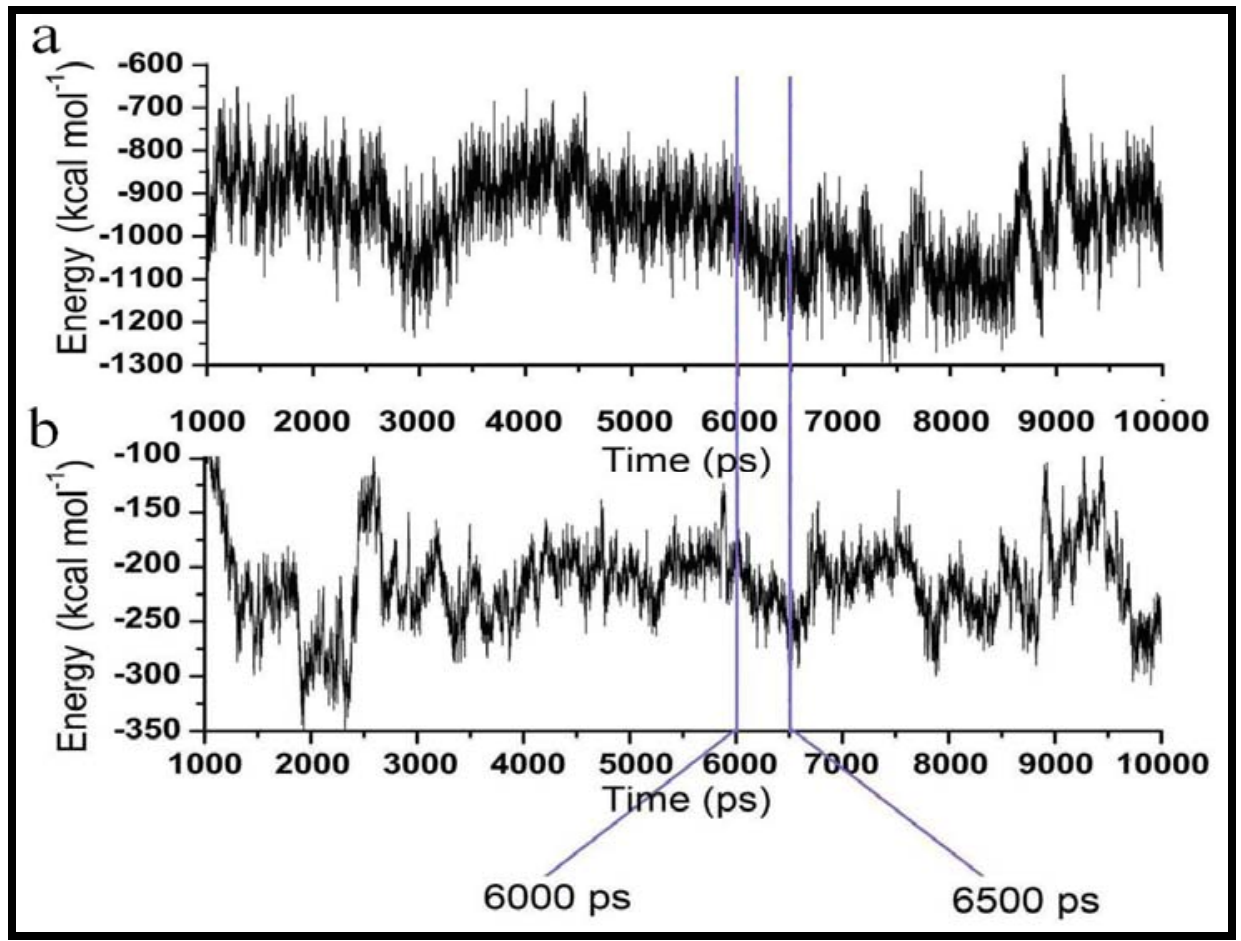

Figure 3: Identification of the primary response of SMSH to the cleavage of the Fe-His bond by energy profiles. (a) The protein potential energy over 10-ns MD simulation. (b) The interaction energy between L2 and other structural elements.

\section{Principal component analysis}

Separating the conformational changes of protein during MD simulation into an essential subspace comprising most of the positional fluctuations are helpful to describe the motions which are important for the function of the protein [14]. The first three eigenvalues of the covariance matrix of $\mathrm{C} \alpha$-displacements over 6-6.5 ns are $17.9,8.3$ and 7.1 , respectively. The first eigenvalue is much larger than the other two. This indicates that the $\mathrm{C} \alpha$ essential motions are along the first eigenvector direction. To further verify the response of SMSH to the cleavage event, we projected the $\mathrm{C} \alpha$ motions onto the first eigenvector which corresponded to the first eigenvalue. Figure 2B shows a superposition of 50 sequential projections of the $\mathrm{C} \alpha$ motions onto the first eigenvector, each separated by 10 ps. The results show that there are no significant $\mathrm{C} \alpha$ motions over the entire protein except L2. This further demonstrates that the information upon the cleavage of the Fe-His bond has been propagated to $\mathrm{L} 2$.

ISSN 0973-2063

Bioinformation 2(7): 296-300 (2008)

\section{Conclusion:}

How the conformational changes are generated and transmitted is of major interest for understanding the allosteric communication mechanism of sGC. The objective of this study was to explore the primary response of the SGC HNOX domain to the cleavage of the Fe-His bond. Based on our results, the energetic conformational transformation of L2 is identified as the primary response of the HNOX domain to the cleavage of the Fe-His bond. The expression of the domains of sGC has suggested that there is a direct interaction between the HNOX domain and the cyclase domain [20]. The activation information from HNOX domain upon the cleavage of the Fe-His bond is possible been propagated to the cyclase domain by the conformational transformation of L2. However, the role of L2 in the communication between the HNOX domain and the cyclase domain remains to be tested further. We expect that our results could facilitate future theory and experimental study on the pathway for transmission 


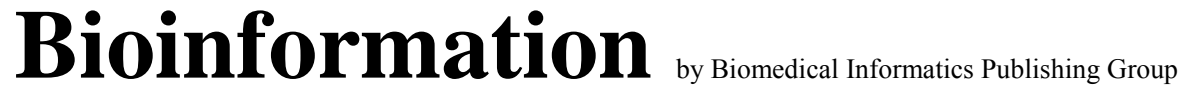

open access

\section{www.bioinformation.net}

\section{Hypothesis}

activation information from the sGC HNOX domain to the cyclase domain.

Acknowledgment:

This work was supported by grants from the National Natural Science Foundation of China (30570404, 30670458).

References:

[01] B. Pal, and T. Kitagawa, J Inorg Biochem., 99: 267 (2005) [PMID: 15598506]

[02] Z. Li et al., Biochemistry, 44: 939 (2005) [PMID: 15654750]

[03] R. Makino et al., J Biol Chem., 278: 11130 (2003) [PMID: 12540839]

[04] Y. Zhao et al., Biochemistry, 37: 4502 (1998) [PMID: 9521770]

[05] J. A. Winger et al., J Biol Chem., 282: 897 (2007) [PMID: 17098738]

[06] L. M. Iyer et al., BMC Genomics, 4: 5 (2003) [PMID: 12590654]

[07] P. Pellicena et al., Proc Natl Acad Sci U S A, 101: 12854 (2004) [PMID: 15326296]

[08] X. Ma et al., Embo J., 26: 578 (2007) [PMID: 17215864]
[09] E. M. Boon et al., J Biol Chem., 28: 21892 (2006) [PMID: 16728401]

[10] C. Notredame et al., J Mol Biol., 302: 205 (2000) [PMID: 10964570]

[11] A. Sali and T. L. Blundell, J Mol Biol., 234: 779 (1993) [PMID: 8254673]

[12] W. Humphrey et al., J Mol Graph, 14: 33 (1996) [PMID: 8744570]

[13] J. C. Phillips et al., J Comput Chem., 26: 1781 (2005) [PMID: 16222654]

[14] A. Amadei et al., Proteins, 17: 412 (1993) [PMID: 8108382]

[15] Y. Gao et al., J Biol Chem., 281: 24637 (2006) [PMID: 16774917]

[16] P. M. Schmidt et al., J Biol Chem., 279: 3025 (2004) [PMID: 14570894]

[17] P. M. Schmidt et al., Eur J Pharmacol., 513: 67 (2005) [PMID: 15878710]

[18] F. Schotte et al., J Struct Biol., 147: 235 (2004) [PMID: 15450293]

[19] A. Sato et al., Proc Natl Acad Sci U S A, 104: 9627 (2007) [PMID: 17517618]

[20] J. A. Winger and M. A. Marletta, Biochemistry, 44: 4083 (2005) [PMID: 15751985]

Edited by P. Kangueane

Citation: Zhang et al., Bioinformation 2(7): 296-300 (2008)

License statement: This is an open-access article, which permits unrestricted use, distribution, and reproduction in any medium, for non-commercial purposes, provided the original author and source are credited. 